\title{
A STUDY TO EXPLORE THE FACTORS ASSOCIATED WITH ANAEMIA AMONG ANTENATAL MOTHERS IN SELECTED HOSPITALS AT ERNAKULAM DISTRICT IN KERALA
}

\author{
Mrs. Bindu T Thomas* \& Dr. Riaz K M** \\ *Research Scholar, Himalayan University, Itanagar, Arunachal Pradesh, India. \\ **Research Guide, Himalayan University, Itanagar, Arunachal Pradesh, India. \\ DOI: http://doi.org/10.47211/tg.2020.v07i03.014
}

\section{ABSTRACT}

The present study, explore the factors associated with anaemia among antenatal mothers at selected hospitals, Ernakulam was undertaken during 2020 in partial fulfillment of the requirement for the Ph. D under Himalayan university. Objective: to explore the factors associated with anemia among antenatal mothers and associate the explorative factors with demographic variables. Design: non experimental descriptive research design. Setting: medical Centre. Sample: 64 antenatal mothers with anemia. Sampling technique: Probability cluster sample. Conceptual Frame Work: Pender's health promotion model (1996). Outcome Measures: Explorative factors of anemia among antenatal mothers. Results: 80 percentages of antenatal mothers belong to medical Centre were having anemia. 48(75 percent) of antenatal mothers were having mild anemia and 16(25 percent) were having moderate anemia. Most of the antenatal mothers with anemia were having poor obstetrical factors related to anemia. Majority of the antenatal mothers were having poor nutrition. Most of the antenatal mothers were not practicing good hygiene and cooking practice. Finding of the study revealed that antenatal mothers have average knowledge related to anemia. There is an association between knowledge with the monthly income of the family, number of children and parity, obstetrical factors with age and monthly income of family, illness factors with age, education, monthly income of family, number of children, parity and food habits, nutritional factors with education and number of children. Conclusion: The result of the study support that there is a positive association between explorative factors of anemia with demographic characteristics. It says the need of mass health teaching to antenatal mothers regarding iron deficiency anemia. Health education was given to antenatal mothers regarding anemia using flash cards.

Key Words: anaemia, antenatal mothers, explorative factors of anemia.

\section{ABOUT AUTHORS:}

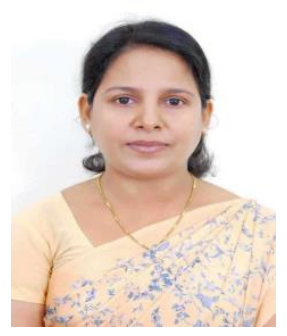

The author Mrs. Bindu T Thomas is research scholar, Himalayan University, Itanagar, Arunachal Pradesh, India.

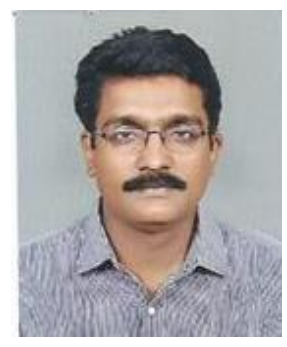

The author Dr. Riaz K M is research supervisor at Himalayan University, Itanagar, Arunachal Pradesh, India. He has presented papers in various national and international conferences. 


\section{INTRODUCTION}

Anaemia, defined as clinical abnormality characterized by reduction in hemoglobin concentration below the normal for age, sex and physiological condition is one of the most worldwide health problem. The prevalence of maternal anemia globally has remained high over the past 30 years. Anaemia, defined as clinical abnormality characterized by reduction in hemoglobin concentration below the normal for age, sex and physiological condition is one of the most worldwide health problem. The prevalence of maternal anemia globally has remained high over the past 30 years. Anemia in pregnancy continues to be a common clinical problem in many developing countries. Approximately 50 percent of women and children in less developed countries are anemic, and 60 percent of the world's anemic population resides in South Asia [Sharma S, 2007]. Anemia is the late manifestation of nutrients needed for hemoglobin synthesis. Most of the anemias are due to inadequate supply of nutrients like iron, folic acid, proteins, amino acids vitamin A, vitamin C and other vitamins of B complex group. In India anemia affects an estimated 50 percent of the population [Helen Keller, 2007]. Pregnancy is one of the most nutritionally demanding times in women's life. Although, it is a normal physiological process, it is a time when the nutritional needs of the mother and the fetus must be met judiciously. Maternal nutrition during pregnancy has a vital influence on the long term prospects of the fetus [Nelson, 2007].

Motherhood should be a time of expectation and joy for a woman, her family and her community. Antenatal care is one of the pillars of safe motherhood interventions that are believed to reduce maternal and perinatal mortality if carried out properly. Universal access to antenatal care is a matter of priority in both developed and developing countries. Antenatal anemia is ranked as the fourth commonest cause of maternal mortality [David P. Urassa, 2002]. A high proportion of women in both industrialized and developing countries become anaemic during pregnancy. Anaemia has a significant impact on the health of the foetus as well as that of the mother.

\section{METHODOLOGY}

\section{Research Approach}

The research approach of this study was exploratory in nature, the investigator seeked to explore the factors associated with anemia among antenatal mothers.

\section{Research Design}

The research design applied for this study was non experimental descriptive research design.

\section{Setting of the Study}

The study was conducted at selected hospitals at Ernakulam District. Every day, approximately 50-100 antenatal mothers are attending the antenatal clinic, during which the high risk mothers are referred to General Hospital, Ernakulam.

\section{Population}

The population comprises of all antenatal mother first visit with hemoglobin below $10 \mathrm{~g} / \mathrm{dl}$

\section{Sample Size}

Probability cluster sampling technique was adapted to select the samples for this study. 64 antenatal mothers who fulfill inclusion criteria were included for the study.

Questionnaire to explore the factors associated with anemia among antenatal mothers

Questionnaire to explore the factors associated with anemia among antenatal mothers include,

\section{$>$ Obstetrical Factors}

This consists of obstetrical factors of antenatal mothers. The scale consists of 10 items. Maximum score is 10 . Each item was scored as, No-0, Yes-1. The total score is classified into poor (score 6 and above), average (score 3-5) and good (score up to 2)

\section{$>$ Illness Factors}

This consists of the illness factors of antenatal mothers. The scale consists of 10 items. Maximum score is 10 . Each item was scored as, N0-0, Yes-1. The total score is classified into poor (score 5 and above), average (score 3-4) and good (score up to 2)

\section{$>$ Nutritional Factors}

Nutritional factors scale consists of the nutrition pattern of anemic mothers. The scale consists of 7 items. Maximum score is 20 . Each item was scored as, Never -0 , rarely -1 , most often -2 , Always -3 . The total score is classified into poor (score up to 3), average (score 4-8) and good (score 9 and above).

\section{$>$ Factors related to cooking practices}




\section{ARTICLES}

This consists of cooking practices of the antenatal mothers. The scale consists of 7 items. Maximum score was 21. Each item was scored as, Always -0 , most often -1 , rarely -2 , Never -3 . The total score is classified into poor (Score up to 4), average (score 5-9), and good (score 10 and above).

\section{$>$ Hygienic factors}

This consists of the hygienic practices of the antenatal mothers. The scale consists of 9 items. Maximum score was 27. Each item was scored as, Never -0 , rarely -1 , most often -2 , Always -3 . The total score is classified into poor (score up to 4), average (score 5 to 9 ) and good (score 10 and above).

\section{STATISTICAL ANALYSIS}

Chi-square was used to find out the association between demographic variables and explorative factors of anemia among antenatal mothers.

\section{DATA ANALYSIS AND INTERPRETATION}

This chapter deals with the description of the samples, analysis and interpretation of the collected data. The data collected is tabulated and the analysis is presented as follows.

Section I: Distribution of samples according to the explorative factors of anaemia among antenatal mothers.

Section II: Association of demographic variable with explorative factors of anaemia.

$>$ Obstetrical factors

$>$ Illness factors

$>$ Nutritional factors of anaemia.

$>$ Cooking practices

$>$ Hygienic practice

\section{Section I}

Distribution of respondents according to the explorative factors of anemia.

\section{Table: 1}

\begin{tabular}{|c|c|c|c|c|c|}
\hline Sl.no & $\begin{array}{l}\text { Explorative } \\
\text { anemia }\end{array}$ & ctors of & $\begin{array}{l}\text { Number } \\
\text { samples }(n=64)\end{array}$ & Percentage (\%) & Mean \\
\hline 1 & $\begin{array}{l}\text { Obstetrical } \\
\text { factors }\end{array}$ & $\begin{array}{l}\text { Poor } \\
\text { Average } \\
\text { Good }\end{array}$ & $\begin{array}{l}5 \\
51 \\
8\end{array}$ & $\begin{array}{l}7.81 \\
79.68 \\
12.5 \\
\end{array}$ & 4.32 \\
\hline 2 & $\begin{array}{l}\text { Illness } \\
\text { factors }\end{array}$ & $\begin{array}{l}\text { Poor } \\
\text { Average } \\
\text { Good }\end{array}$ & $\begin{array}{l}14 \\
39 \\
11 \\
\end{array}$ & $\begin{array}{l}21.88 \\
60.94 \\
17.19 \\
\end{array}$ & 3.17 \\
\hline 3 & $\begin{array}{l}\text { Nutritional } \\
\text { factors }\end{array}$ & $\begin{array}{l}\text { Poor } \\
\text { Average } \\
\text { Good }\end{array}$ & $\begin{array}{l}17 \\
22 \\
25\end{array}$ & $\begin{array}{l}26.56 \\
34.38 \\
39.06 \\
\end{array}$ & 6.20 \\
\hline 4 & $\begin{array}{l}\text { Cooking } \\
\text { practices }\end{array}$ & $\begin{array}{l}\text { Poor } \\
\text { Average } \\
\text { Good }\end{array}$ & $\begin{array}{l}27 \\
22 \\
15\end{array}$ & $\begin{array}{l}42.18 \\
34.38 \\
23.44 \\
\end{array}$ & 7.42 \\
\hline 5 & $\begin{array}{l}\text { Hygienic } \\
\text { factors }\end{array}$ & $\begin{array}{l}\text { Poor } \\
\text { Average } \\
\text { Good }\end{array}$ & $\begin{array}{l}24 \\
24 \\
26\end{array}$ & $\begin{array}{l}37.5 \\
37.5 \\
25\end{array}$ & 7.81 \\
\hline
\end{tabular}




\section{ARTICLES}

\section{SECTION -II}

Association of demographic variables with explorative factors of anaemia among antenatal mothers.

Table 2 Association of demographic variables with obstetrical factors

\begin{tabular}{|c|c|c|c|c|c|c|c|}
\hline \multirow[t]{2}{*}{ SI. No. } & \multicolumn{2}{|c|}{ Demographic variables } & \multicolumn{2}{|c|}{ Obstetrical factors $(n=64)$} & \multirow[b]{2}{*}{ Good } & \multirow[t]{2}{*}{ df } & \multirow[t]{2}{*}{$x^{2}$} \\
\hline & & & poor & average & & & \\
\hline 1 & Age in years & $\begin{array}{l}\text { Upto } 20 \\
21-25 \\
26-30 \\
31-35 \\
36-40\end{array}$ & $\begin{array}{l}3 \\
1 \\
1 \\
0 \\
0\end{array}$ & $\begin{array}{l}4 \\
10 \\
13 \\
21 \\
3\end{array}$ & $\begin{array}{l}0 \\
2 \\
1 \\
4 \\
1\end{array}$ & 8 & $15.60 * *$ \\
\hline 2 & education & $\begin{array}{l}\text { Illiterate } \\
\text { Primary } \\
\text { Secondary } \\
\text { Graduate }\end{array}$ & $\begin{array}{l}1 \\
2 \\
1 \\
1\end{array}$ & $\begin{array}{l}22 \\
15 \\
9 \\
5\end{array}$ & $\begin{array}{l}3 \\
2 \\
1 \\
2\end{array}$ & 6 & 2.61 (NS) \\
\hline 3 & religion & $\begin{array}{l}\text { Hindu } \\
\text { Christian }\end{array}$ & \begin{tabular}{|l}
4 \\
1
\end{tabular} & $\begin{array}{l}46 \\
5\end{array}$ & $\begin{array}{l}6 \\
2\end{array}$ & 2 & 1.72 (NS) \\
\hline 4 & occupation & $\begin{array}{l}\text { Home maker } \\
\text { Coolie }\end{array}$ & $\begin{array}{l}4 \\
1 \\
\end{array}$ & $\begin{array}{l}42 \\
9\end{array}$ & $\begin{array}{l}6 \\
2\end{array}$ & 2 & $.28(\mathrm{NS})$ \\
\hline 5 & $\begin{array}{l}\text { Monthly } \\
\text { income }\end{array}$ & $\begin{array}{l}<1000 \\
1001-1500 \\
>2000 \\
\end{array}$ & $\begin{array}{l}2 \\
3 \\
0 \\
\end{array}$ & $\begin{array}{l}46 \\
2 \\
3 \\
\end{array}$ & $\begin{array}{l}5 \\
2 \\
1 \\
\end{array}$ & 4 & $17.34^{* *}$ \\
\hline 6 & Type of family & $\begin{array}{l}\text { Nuclear } \\
\text { joint }\end{array}$ & $\begin{array}{l}4 \\
1\end{array}$ & $\begin{array}{l}47 \\
4\end{array}$ & $\begin{array}{l}5 \\
3\end{array}$ & 2 & 5.91 (NS) \\
\hline 7 & No. of children & $\begin{array}{l}\text { No child } \\
\text { One child } \\
\text { Two and } \\
\text { above } \\
\begin{array}{l}\text { Five } \\
\text { above }\end{array} \\
\end{array}$ & $\begin{array}{l}1 \\
2 \\
1 \\
1\end{array}$ & $\begin{array}{l}8 \\
7 \\
33 \\
3\end{array}$ & $\begin{array}{l}2 \\
2 \\
2 \\
2\end{array}$ & 6 & 9.17(NS) \\
\hline 8 & parity & $\begin{array}{l}\text { Primi gravida } \\
\text { Multi gravida } \\
\text { Grand } \\
\text { multigravida }\end{array}$ & $\begin{array}{l}1 \\
3 \\
1\end{array}$ & $\begin{array}{l}8 \\
40 \\
3\end{array}$ & $\begin{array}{l}2 \\
4 \\
2\end{array}$ & 4 & 5.14(NS) \\
\hline 9 & Food habits & $\begin{array}{l}\text { Vegetarian } \\
\text { Non } \\
\text { vegetarian }\end{array}$ & $\begin{array}{l}1 \\
4\end{array}$ & $\begin{array}{l}5 \\
46\end{array}$ & $\begin{array}{l}7 \\
1\end{array}$ & 2 & $.51(\mathrm{NS})$ \\
\hline
\end{tabular}

**Significant at 0.01 level NS - Not Significant

Chi square analysis at 0.01 level of significance shows there is an association between obstetrical factors with age and monthly income of the family. There is no association between other demographic variable with obstetrical factors. 


\section{ARTICLES}

Table -3 Associations of demographic variables with illness factors

\begin{tabular}{|c|c|c|c|c|c|c|c|}
\hline \multirow[t]{2}{*}{ SI. No. } & \multicolumn{2}{|c|}{ Demographic variables } & \multicolumn{2}{|c|}{ Illness factors( $n=64)$} & \multirow[b]{2}{*}{ Good } & \multirow[t]{2}{*}{ df } & \multirow[t]{2}{*}{$\mathrm{x}^{2}$} \\
\hline & & & poor & average & & & \\
\hline 1 & Age in years & $\begin{array}{l}\text { Up to } 20 \\
21-25 \\
26-30 \\
31-35 \\
36-40\end{array}$ & $\begin{array}{l}5 \\
8 \\
1 \\
0 \\
0\end{array}$ & $\begin{array}{l}2 \\
4 \\
9 \\
21 \\
3\end{array}$ & $\begin{array}{l}0 \\
1 \\
5 \\
4 \\
1\end{array}$ & 8 & $42.85 * *$ \\
\hline 2 & education & $\begin{array}{l}\text { Illiterate } \\
\text { Primary } \\
\text { Secondary } \\
\text { Graduate } \\
\end{array}$ & \begin{tabular}{|l}
0 \\
3 \\
4 \\
4 \\
\end{tabular} & $\begin{array}{l}11 \\
8 \\
6 \\
3 \\
\end{array}$ & $\begin{array}{l}15 \\
8 \\
1 \\
1 \\
\end{array}$ & 6 & $17.68^{* *}$ \\
\hline 3 & religion & $\begin{array}{l}\text { Hindu } \\
\text { Christian }\end{array}$ & \begin{tabular}{|l|}
10 \\
4 \\
\end{tabular} & $\begin{array}{l}36 \\
3\end{array}$ & $\begin{array}{l}10 \\
1\end{array}$ & 2 & $\begin{array}{l}4.26 \\
\text { (NS) }\end{array}$ \\
\hline 4 & occupation & $\begin{array}{l}\text { Home maker } \\
\text { Coolie }\end{array}$ & $\begin{array}{l}13 \\
1\end{array}$ & $\begin{array}{l}31 \\
8\end{array}$ & $\begin{array}{l}9 \\
2\end{array}$ & 2 & $\begin{array}{l}1.31 \\
\text { (NS) }\end{array}$ \\
\hline 5 & $\begin{array}{l}\text { Monthly } \\
\text { income }\end{array}$ & $\begin{array}{l}<1000 \\
1001-1500 \\
>2000\end{array}$ & $\begin{array}{l}7 \\
4 \\
3\end{array}$ & $\begin{array}{l}36 \\
2 \\
1\end{array}$ & $\begin{array}{l}10 \\
1 \\
0\end{array}$ & 4 & $14.1^{* *}$ \\
\hline 6 & Type of family & $\begin{array}{l}\text { Nuclear } \\
\text { joint }\end{array}$ & $\begin{array}{l}10 \\
4 \\
\end{array}$ & $\begin{array}{l}37 \\
2\end{array}$ & $\begin{array}{l}9 \\
2\end{array}$ & 2 & $\begin{array}{l}5.59 \\
\text { (NS) }\end{array}$ \\
\hline 7 & No. of children & $\begin{array}{l}\text { No child } \\
\text { One child } \\
\text { Two and } \\
\text { above } \\
\begin{array}{l}\text { Five and } \\
\text { above }\end{array} \\
\end{array}$ & $\begin{array}{l}7 \\
6 \\
1 \\
0\end{array}$ & $\begin{array}{l}4 \\
4 \\
30 \\
1\end{array}$ & $\begin{array}{l}0 \\
1 \\
5 \\
5\end{array}$ & 6 & $46.68^{* *}$ \\
\hline 8 & parity & $\begin{array}{l}\text { Primi gravida } \\
\text { Multi gravida } \\
\text { Grand } \\
\text { multigravida }\end{array}$ & $\begin{array}{l}7 \\
7 \\
0\end{array}$ & $\begin{array}{l}4 \\
34 \\
1\end{array}$ & $\begin{array}{l}0 \\
6 \\
5\end{array}$ & 4 & $33.09 * *$ \\
\hline 9 & Food habits & $\begin{array}{l}\text { Vegetarian } \\
\text { Non } \\
\text { vegetarian }\end{array}$ & $\begin{array}{l}5 \\
9\end{array}$ & $\begin{array}{l}2 \\
37\end{array}$ & $\begin{array}{l}0 \\
11\end{array}$ & 2 & $11.55^{* *}$ \\
\hline
\end{tabular}

** Significant at 0.01 level NS- not significant

Chi-square analysis at 0.01 level of significance shows there is an association between illness factors with age, education, monthly income of family, number of children, parity and food habits. There is no association between illness factors with other demographic variables. 


\section{ARTICLES}

Table-4 association of demographic variables with

nutritional factors

\begin{tabular}{|c|c|c|c|c|c|c|c|}
\hline \multirow[t]{2}{*}{ SI. No. } & \multicolumn{2}{|c|}{ Demographic variables } & \multicolumn{2}{|c|}{ Nutritional factors $(n=64)$} & \multirow[b]{2}{*}{ Good } & \multirow[t]{2}{*}{ df } & \multirow[t]{2}{*}{$x^{2}$} \\
\hline & & & poor & average & & & \\
\hline 1 & Age in years & $\begin{array}{l}\text { Up to } 20 \\
21-25 \\
26-30 \\
31-35 \\
36-40 \\
\end{array}$ & $\begin{array}{l}3 \\
4 \\
2 \\
10 \\
2 \\
\end{array}$ & $\begin{array}{l}2 \\
6 \\
6 \\
8 \\
1\end{array}$ & $\begin{array}{l}2 \\
3 \\
7 \\
7 \\
1\end{array}$ & 8 & $\begin{array}{l}12.17 \\
\text { (NS) }\end{array}$ \\
\hline 2 & Education & $\begin{array}{l}\text { Illiterate } \\
\text { Primary } \\
\text { Secondary } \\
\text { Graduate }\end{array}$ & $\begin{array}{l}10 \\
9 \\
2 \\
0\end{array}$ & $\begin{array}{l}10 \\
8 \\
3 \\
2 \\
\end{array}$ & $\begin{array}{l}6 \\
2 \\
6 \\
6\end{array}$ & 6 & $15.58 * *$ \\
\hline 3 & religion & $\begin{array}{l}\text { Hindu } \\
\text { Christian }\end{array}$ & $\begin{array}{l}19 \\
2 \\
\end{array}$ & $\begin{array}{l}19 \\
4 \\
\end{array}$ & $\begin{array}{l}18 \\
2 \\
\end{array}$ & 2 & $\begin{array}{l}0.8 \\
\text { (NS) }\end{array}$ \\
\hline 4 & Occupation & $\begin{array}{l}\text { Home maker } \\
\text { Coolie }\end{array}$ & $\begin{array}{l}15 \\
6 \\
\end{array}$ & $\begin{array}{l}19 \\
4 \\
\end{array}$ & $\begin{array}{l}18 \\
2 \\
\end{array}$ & 2 & $\begin{array}{l}2.58 \\
\text { (NS) }\end{array}$ \\
\hline 5 & $\begin{array}{l}\text { Monthly } \\
\text { income }\end{array}$ & $\begin{array}{l}<1000 \\
1001-1500 \\
>2000\end{array}$ & $\begin{array}{l}19 \\
2 \\
0 \\
\end{array}$ & $\begin{array}{l}18 \\
3 \\
2 \\
\end{array}$ & $\begin{array}{l}16 \\
2 \\
2 \\
\end{array}$ & 4 & $\begin{array}{l}2.36 \\
\text { (NS) }\end{array}$ \\
\hline 6 & $\begin{array}{l}\text { Type of } \\
\text { family }\end{array}$ & $\begin{array}{l}\text { Nuclear } \\
\text { joint }\end{array}$ & $\begin{array}{l}19 \\
2 \\
\end{array}$ & $\begin{array}{l}22 \\
1 \\
\end{array}$ & $\begin{array}{l}15 \\
5 \\
\end{array}$ & 2 & $\begin{array}{l}4.45 \\
\text { (NS) }\end{array}$ \\
\hline 7 & $\begin{array}{l}\text { No. } \\
\text { children }\end{array}$ & $\begin{array}{ll}\text { No child } & \\
\text { One child } & \\
\text { Two } & \text { and } \\
\text { above } & \\
\text { Five } & \text { and } \\
\text { above } & \\
\end{array}$ & $\begin{array}{l}3 \\
2 \\
12 \\
4\end{array}$ & $\begin{array}{l}4 \\
5 \\
13 \\
1\end{array}$ & $\begin{array}{l}4 \\
4 \\
11 \\
\\
1\end{array}$ & 6 & $\begin{array}{l}4.43 \\
\text { (NS) }\end{array}$ \\
\hline 8 & parity & $\begin{array}{l}\text { Primi gravida } \\
\text { Multi gravida } \\
\text { Grand } \\
\text { multigravida }\end{array}$ & $\begin{array}{l}3 \\
14 \\
4\end{array}$ & $\begin{array}{l}4 \\
18 \\
1\end{array}$ & \begin{tabular}{|l|}
4 \\
15 \\
1
\end{tabular} & 4 & $\begin{array}{l}3.54 \\
\text { (NS) }\end{array}$ \\
\hline 9 & Food habits & $\begin{array}{l}\text { Vegetarian } \\
\text { Non } \\
\text { vegetarian }\end{array}$ & $\begin{array}{l}3 \\
18\end{array}$ & $\begin{array}{l}2 \\
21\end{array}$ & $\begin{array}{l}2 \\
18\end{array}$ & 2 & $\begin{array}{l}0.37 \\
\text { (NS) }\end{array}$ \\
\hline
\end{tabular}

**Significant at $\mathbf{0 . 0 1} \quad$ NS- not significant

Chi-square analysis at 0.01 level of significance shows there is an association between nutritional factors with education. There is no association between nutritional factors with other demographic variables. 


\section{ARTICLES}

Table-5 association of demographic variables with cooking practices

\begin{tabular}{|c|c|c|c|c|c|c|c|}
\hline \multirow{3}{*}{$\begin{array}{l}\text { SI. No. } \\
\\
1\end{array}$} & \multirow{2}{*}{\multicolumn{2}{|c|}{ Demographic variables }} & \multicolumn{2}{|c|}{ Cooking practices $(n=64)$} & \multirow[b]{2}{*}{ Good } & \multirow[t]{2}{*}{ df } & \multirow[t]{2}{*}{$x^{2}$} \\
\hline & & & poor & average & & & \\
\hline & Age in years & $\begin{array}{l}\text { Up to } 20 \\
21-25 \\
26-30 \\
31-35 \\
36-40\end{array}$ & $\begin{array}{l}5 \\
4 \\
4 \\
11 \\
3\end{array}$ & $\begin{array}{l}1 \\
3 \\
7 \\
10 \\
1 \\
\end{array}$ & $\begin{array}{l}1 \\
6 \\
4 \\
4 \\
0\end{array}$ & 8 & $\begin{array}{l}10.69 \\
\text { (NS) }\end{array}$ \\
\hline 2 & Education & $\begin{array}{l}\text { Illiterate } \\
\text { Primary } \\
\text { Secondary } \\
\text { Graduate }\end{array}$ & $\begin{array}{l}20 \\
7 \\
0 \\
0 \\
\end{array}$ & $\begin{array}{l}4 \\
8 \\
7 \\
3\end{array}$ & $\begin{array}{l}2 \\
4 \\
4 \\
5\end{array}$ & 6 & $30.17 * *$ \\
\hline 3 & religion & $\begin{array}{l}\text { Hindu } \\
\text { Christian }\end{array}$ & $\begin{array}{l}23 \\
4\end{array}$ & $\begin{array}{l}19 \\
3\end{array}$ & $\begin{array}{l}14 \\
1\end{array}$ & 2 & $\begin{array}{l}0.2 \\
\text { (NS) }\end{array}$ \\
\hline 4 & Occupation & $\begin{array}{l}\text { Home maker } \\
\text { Coolie }\end{array}$ & $\begin{array}{l}19 \\
8 \\
\end{array}$ & $\begin{array}{l}19 \\
3 \\
\end{array}$ & $\begin{array}{l}14 \\
1 \\
\end{array}$ & 2 & $\begin{array}{l}3.89 \\
\text { (NS) }\end{array}$ \\
\hline 5 & Monthly income & $\begin{array}{l}<1000 \\
1001-1500 \\
>2000\end{array}$ & $\begin{array}{l}24 \\
3 \\
0 \\
\end{array}$ & $\begin{array}{l}17 \\
3 \\
2 \\
\end{array}$ & $\begin{array}{l}12 \\
1 \\
2 \\
\end{array}$ & 4 & $\begin{array}{l}6.06 \\
\text { (NS) }\end{array}$ \\
\hline 6 & Type of family & $\begin{array}{l}\text { Nuclear } \\
\text { joint }\end{array}$ & $\begin{array}{l}26 \\
1\end{array}$ & $\begin{array}{l}17 \\
5\end{array}$ & $\begin{array}{l}13 \\
2\end{array}$ & 2 & $\begin{array}{l}4.03 \\
\text { (NS) }\end{array}$ \\
\hline 7 & No. of children & $\begin{array}{l}\text { No child } \\
\text { One child } \\
\text { Two and } \\
\text { above } \\
\text { Five and } \\
\text { above }\end{array}$ & $\begin{array}{l}3 \\
1 \\
19 \\
4\end{array}$ & $\begin{array}{l}4 \\
6 \\
10 \\
2\end{array}$ & $\begin{array}{l}4 \\
4 \\
7 \\
0\end{array}$ & 6 & $\begin{array}{l}10.23 \\
\text { (NS) }\end{array}$ \\
\hline 8 & parity & $\begin{array}{l}\text { Primi } \\
\text { gravida } \\
\text { Multi } \\
\text { gravida } \\
\text { Grand multi } \\
\text { gravida }\end{array}$ & $\begin{array}{l}3 \\
20 \\
4\end{array}$ & $\begin{array}{l}4 \\
16 \\
2\end{array}$ & $\begin{array}{l}4 \\
11 \\
0\end{array}$ & 4 & $\begin{array}{l}5.10 \\
\text { (NS) }\end{array}$ \\
\hline 9 & Food habits & $\begin{array}{l}\text { Vegetarian } \\
\text { Non } \\
\text { vegetarian }\end{array}$ & $\begin{array}{l}4 \\
23 \\
\end{array}$ & $\begin{array}{l}2 \\
20 \\
\end{array}$ & $\begin{array}{l}1 \\
14 \\
\end{array}$ & 2 & 9.06 \\
\hline
\end{tabular}

\footnotetext{
**Significant at $0.01 \quad$ NS- not significant
}

Chi-square analysis at 0.01 level of significance shows there is an association between cooking practices with education and food habits. There is no association between cooking practices with other demographic variables. 


\section{ARTICLES}

Table-6 association of demographic variable with hygienic factors

\begin{tabular}{|c|c|c|c|c|c|c|c|}
\hline \multirow[t]{2}{*}{ SI. No. } & \multicolumn{2}{|c|}{ Demographic variables } & \multicolumn{2}{|c|}{ Hygienic factors $(n=64)$} & \multirow[b]{2}{*}{ Good } & \multirow[t]{2}{*}{ Df } & \multirow[t]{2}{*}{$x^{2}$} \\
\hline & & & Poor & average & & & \\
\hline 1 & Age in years & $\begin{array}{l}\text { Up to } 20 \\
21-25 \\
26-30 \\
31-35 \\
36-40\end{array}$ & $\begin{array}{l}5 \\
4 \\
4 \\
11 \\
3\end{array}$ & $\begin{array}{l}1 \\
3 \\
7 \\
10 \\
1\end{array}$ & $\begin{array}{l}1 \\
6 \\
4 \\
4 \\
0\end{array}$ & 8 & $\begin{array}{l}10.69 \\
\text { (NS) }\end{array}$ \\
\hline 2 & Education & $\begin{array}{l}\text { Illiterate } \\
\text { Primary } \\
\text { Secondary } \\
\text { Graduate }\end{array}$ & $\begin{array}{l}20 \\
7 \\
0 \\
0\end{array}$ & $\begin{array}{l}4 \\
8 \\
7 \\
3\end{array}$ & $\begin{array}{l}2 \\
4 \\
4 \\
5\end{array}$ & 6 & $30.1^{* *}$ \\
\hline 3 & religion & $\begin{array}{l}\text { Hindu } \\
\text { Christian }\end{array}$ & $\begin{array}{l}23 \\
4 \\
\end{array}$ & $\begin{array}{l}19 \\
3 \\
\end{array}$ & $\begin{array}{l}14 \\
1 \\
\end{array}$ & 2 & $\begin{array}{l}0.2 \\
\text { (NS) }\end{array}$ \\
\hline 4 & Occupation & $\begin{array}{l}\text { Home maker } \\
\text { Coolie }\end{array}$ & $\begin{array}{l}19 \\
8 \\
\end{array}$ & $\begin{array}{l}19 \\
3 \\
\end{array}$ & $\begin{array}{l}14 \\
1 \\
\end{array}$ & 2 & $\begin{array}{l}3.89 \\
\text { (NS) }\end{array}$ \\
\hline 5 & Monthly income & $\begin{array}{l}<1000 \\
1001-1500 \\
>2000 \\
\end{array}$ & $\begin{array}{l}24 \\
3 \\
0 \\
\end{array}$ & $\begin{array}{l}17 \\
3 \\
2 \\
\end{array}$ & $\begin{array}{l}12 \\
1 \\
2 \\
\end{array}$ & 4 & $\begin{array}{l}6.06 \\
\text { (NS) }\end{array}$ \\
\hline 6 & Type of family & $\begin{array}{l}\text { Nuclear } \\
\text { Joint }\end{array}$ & $\begin{array}{l}26 \\
1 \\
\end{array}$ & $\begin{array}{l}17 \\
5 \\
\end{array}$ & $\begin{array}{l}13 \\
2 \\
\end{array}$ & 2 & $\begin{array}{l}4.03 \\
\text { (NS) }\end{array}$ \\
\hline 7 & No. of children & $\begin{array}{l}\text { No child } \\
\text { One child } \\
\text { Two and } \\
\text { above } \\
\begin{array}{l}\text { Five and } \\
\text { above }\end{array}\end{array}$ & $\begin{array}{l}3 \\
1 \\
19 \\
4\end{array}$ & $\begin{array}{l}4 \\
6 \\
10 \\
2\end{array}$ & $\begin{array}{l}4 \\
4 \\
7 \\
\\
0\end{array}$ & 6 & $10.23^{* *}$ \\
\hline 8 & parity & $\begin{array}{l}\text { Primi } \\
\text { gravida } \\
\text { Multi } \\
\text { gravida } \\
\text { Grand multi } \\
\text { gravida }\end{array}$ & $\begin{array}{l}3 \\
20 \\
4\end{array}$ & $\begin{array}{l}4 \\
16 \\
2\end{array}$ & $\begin{array}{l}4 \\
11 \\
0\end{array}$ & 4 & $\begin{array}{l}5.10 \\
\text { (NS) }\end{array}$ \\
\hline 9 & Food habits & $\begin{array}{l}\text { Vegetarian } \\
\text { Non } \\
\text { vegetarian }\end{array}$ & $\begin{array}{l}4 \\
23\end{array}$ & $\begin{array}{l}2 \\
20\end{array}$ & $\begin{array}{l}1 \\
14\end{array}$ & 2 & $\begin{array}{l}9.06 \\
\text { (NS) }\end{array}$ \\
\hline
\end{tabular}

**Significant at $\mathbf{0 . 0 1}$ NS- not significant

Chi-square analysis at 0.01 level of significance shows there is an association between hygienic factors with education and number of children. There is no association between hygienic factors with other demographic variables. 


\section{DISCUSSION}

Motherhood should be a time of expectations and joy for a woman, her family and community. For women in developing countries, however, the reality of motherhood is often grim. For these women, motherhood is often marred by unforeseen complications of pregnancy and child birth. Some die in the prime period of their lives and in great distress. The data of the study were analyzed statistically and discussed below.

Associate demographic variable with explorative factors of anemia among antenatal mothers

$>$ Associate demographic variable with obstetrical factors

Chi - square analysis at 0.01 level of significance showed that there is an association between obstetrical factors with age and monthly income of the family.

$>$ Associate demographic variable with illness factors

Chi - Square analysis at 0.01 level of significant showed that there is an association between illness factors with age, education, monthly income of the family, number of children, parity and food habits.

$>$ Associate demographic variable with nutritional factors

Chi - square analysis at 0.01 level of significant showed that there is an association between nutritional factors with education.

$>$ Associate demographic variable with cooking practices

Chi-square analysis at 0.01 level of significant showed that there is an association between cooking practices with education and food habits.

$>$ Associate demographic variable with hygienic practices

Chi - square analysis at 0.01 level of significant showed that there is an association between hygienic practices with education and number of children.

The tool used for data collection consists of questionnaire such as Explorative factors of anemia including obstetrical, illness, nutritional, cooking practices, and hygienic factors. The data were collected for a period of one month. Descriptive and inferential statistics were used in statistical analysis. Chi - square was used to find out the association between demographic variable with knowledge of antenatal mothers and to associate demographic variable with explorative factors of anemia among antenatal mothers.

\section{CONCLUSION}

The study tested and accepted the hypothesis that, there is a positive association between explorative factors such as obstetrical, illness, nutritional, cooking and hygienic factors to anemia among antenatal mothers. If mothers knowledge regarding anemia is improved the risk of pregnancy related anemia can be prevented. Nurses caring for antenatal mothers need to become increasingly more involved in conducting community assessments that focus specifically on antenatal mothers with high risk pregnancy. The data obtained from this type of assessments can be used for preventing anemia among antenatal mothers at all levels.

\section{REFERENCE}

1. Agarwal 'A.K. et al' (1999). Prevalence of anemia during pregnancy in district Burdwan, West Bengal, Indian Journal of public health. 4(1), pp. 40-45.

2. Dash B. B. (2007). Burden of chronic severe anemia in pregnancy, Obstetrics and Gymea today, pp. 26-30

3. David. P Urassa. (2002). Child Survival and Safe Motherhood, Journal of Community Medicine, 2(1), pp. 23-26.

4. Helen Keller. (2007). Complications of anemia in pregnancy Obstetrics and Gynae today, pp. 40-43.

5. Nelson M. (2007). Adolescence and anemia, Indian Journal of Medical Research, pp35-39.

6. Raina N. (2007). Anemia in pregnancy, Obstetrics and Gynae today, pp.50-55.

7. Sharma K. (2002). Prevalence of anemia in India, Indian Journal of Community Medicine, PP.48-53.

8. Sharma S. (2007). Prevalence of anemia and its effect in pregnancy, Obstetrics and Gynae today, pp. 30-35.

9. Varma A .et al. (2004). Importance of iron supplementation in pregnanacy. American Journal of Public Health. pp. 40-45. 